\title{
Star clusters and single stellar populations
}

\author{
Licai Deng ${ }^{1}$ and Yu Xin ${ }^{2}$ \\ ${ }^{1}$ National Astronomical Observatories, Chinese Academy of Sciences, \\ Beijing 100012, China \\ email: licai@bao.ac.cn \\ ${ }^{2}$ Argelander Institute for Astronomy, Unversity of Bonn, \\ 53121 Bonn, Germany \\ email: yxin@astro.uni-bonn.de
}

\begin{abstract}
Star clusters are ideal laboratories to test the theory of stellar evolution and provide very tight constraints on the concept of single stellar poputions (SSPs). Observations show that some stars fail to conform to the theoretical evolutionary scenario applicable to single stars. These special objects, particularly blue stragglers, present a challenge to our current theory of stellar evolution. They may be very important in the context of the integrated spectral properties of clusters. Here, we review the construction of SSP models, both empirically using star clusters and theoretically based on binary-interaction theory.
\end{abstract}

Keywords. blue stragglers, open clusters and associations: general, Galaxy: stellar content

\section{Introduction}

Simple stellar population (SSP) theory provides a very effective way to study the stellar content in complicated, unresolved stellar systems. By definition, an SSP is a group of stars born at the same time from the same gas cloud, so that they have the same chemical composition at the time of starbirth and the same age. The masses of the SSP's member stars are given by a certain power-law mass distribution at the time of their birth. By implementing the theory of stellar evolution (for single stars) and stellar atmosphere models, the population's behavior during evolution and its observable quantities such as the integrated spectrum, photometric colors, mass-to-light ratio, etc., can be predicted. This technique is known as evolutionary population synthesis (EPS; Bressan et al. 1994; Bertelli et al. 1994; Worthy 1994; Bruzual \& Charlot 2003, hereafter BC03). However, the central engine driving EPS, the theory of stellar evolution, does not cover all observational evidence, even for those stellar populations resolved on colormagnitude diagrams (CMDs) of star clusters. The blue stragglers (BSs), first discovered in the globular cluster M3 (Sandage 1953) in the Galaxy, and later proved common in all resolved stellar systems, for instance, cannot be explained directly. As they are thoroughly studied in the well-known Galactic clusters, BSs cannot be excluded from the clusters' stellar populations, but they cannot be descendants of single stars. Therefore, they have to be accounted for in some way other than through single-star evolution theory. BSs are now understood as the remnants of stellar interactions, including mass exchange (Tian et al. 2006; Chen \& Han 2009), coalescence of close binary systems and collisional encounters in dense environments (Sills et al. 2005; Ferraro et al. 2003). These processes happen in the real world and certainly affect the general properties of SSPs. They therefore introduce some uncertainties into applications of EPS.

SSP models can also be built by direct observations of star clusters. This technique is referred to as 'optimized population synthesis' (e.g., Bica \& Alloin 1986a,b). It optimizes the problems of the stellar initial mass function and stellar atmospheres. However, due 
to limitations of parameter-space coverage and issues to do with observations such as sampling uncertainties, it has fewer applications to studies of galaxies compared with EPS.

Assessment of BSs in terms of their contributions to their host population can be realized either theoretically or empirically. In principle, by theoretically collating all physics relevant to stellar interactions and assembling the results into the conventional SSP framework, one could construct more realistic SSP models. However, because the various combinations of binary parameters and interacting processes are usually too complicated, the results are not sufficiently convincing. In fact, the formation of BSs is much more complicated and stochastic than can be dealt with by existing theoretical models. This is evident from the distributions of BSs in star cluster CMDs. Therefore, empirical treatment seems a better choice. By building up synthetic SSP models based on star clusters covering large grids in both age and metallicity, one can make a model database available for EPS similar to that of the conventional SSP grids. Empirical models constructed this way can be used as an observational constraint to theoretical studies of SSP models and the stellar-interaction processes included in the models.

Here, we analyze the uncertainties in the conventional SSP models due to BSs, using empirical models based on our Galactic open cluster (OC) sample (Deng et al. 1999; Xin \& Deng 2005-hereafter XD05-Xin et al. 2006, hereafter XDH06), and then compare the results with theoretical binary-synthesis models.

\section{Observational facts}

Since the first identification of BSs by Sandage (1953) in his work on the globular cluster M3, BSs have been found to be a common ingredient of resolved stellar systems on various scales, from star clusters to galaxies. BSs in star clusters can be clearly identified in cluster CMDs based on photometric observations. As BSs do not obey the best-fitting cluster isochrones in a given CMD, determining whether or not they are cluster members is crucial. For most Galactic globular clusters, membership is not of special concern, simply because field contamination is not a big problem. For OCs located in the disk and therefore surrounded by diffusely distributed field stars, however, membership is an important issue. This problem can be handled with membership information from propermotion and/or radial velocity observations. For our analysis, we will rely on cataloged clusters with relatively robust information on their stellar membership (Ahumada \& Lapasset 1995; Dias et al. 2002).

In principle, Galactic GCs can also be used to study SSP models. The BS contents in these clusters have been made available using HST observations by Piotto et al. (2004). However, considering the general (diffuse) stellar content of galaxies, the conditions related to the formation mechanisms of BSs (e.g., binary fractions, binary parameter distributions, dynamical evolution of the system, overall stellar density and collision rate, and its evolution within the system, etc.) will likely be more like those in OCs than in GCs. For this reason, we will focus on OCs.

\section{Results from open clusters}

Based on the photometric catalog of BSs in OCs (Ahamada \& Lapasset 1995), we have constructed synthetic integrated spectral-energy distributions (ISEDs) for selected clusters in a series of recent papers (Deng et al. 1999; XD05; XDH06). The underlying ideas, assumptions and our approach are as follows. 
- Assuming that OCs can be approximated as natural SSPs, we first restore the contributions of the evaporated low-mass stars (not important in terms of their integrated light, but still considered for reasons of consistency). This can be realized by substituting the observed stellar component by a conventional SSP model isochrone. All stars in the population obey a single initial mass function (assumed to be Salpeter-like), including stars that eventually evolve into BSs through any formation channel.

- We assume that the formation of BSs will not change the mass function of the cluster. Considering the number of BSs (usually of order 10) and the total number of stars (of order 1000), this is a good approximation.

- We also assume that BSs are basically hydrogen-burning stars, so that their fundamental physical parameters can be determined by fitting them using main-sequence models. According to our present understanding of BSs, this assumption is quite secure. This can also be justified by spectrophotometric observations of BSs, as done in, e.g., NGC 2682 (M67; Liu et al. 2008).

- All other faint 'straggling' stars, including BSs below the turnoff and underlopers below the subgiant branch, are neglected in constructing the synthetic SSP model. This is justified by the fact that the total number of such objects is usually very small, and they are not important when considering the integrated light.

After taking all these assumptions into account, the synthetic SSP model for a cluster can be constructed based on two components. The first is composed of all stars represented by the best-fitting isochrone in the CMD and all other stars evaporated dynamically during the past evolutionary history of the cluster. This is nothing but the conventional SSP model. The second component includes the BSs; bright stragglers at redder colors (i.e., yellow or red stragglers) are also taken into account if they exist in the catalog. For the second component (the 'BS component'), the spectral properties are derived by fitting single-star models to the individual objects using the algorithm presented in XD05. The sum of all light from the members making up this component provides us with the final modifications to the conventional SSP model.

As a result, the synthetic ISEDs of stellar populations are drastically different from the conventional SSP models for a given age and metallicity. In terms of the shape of the spectral properties, the synthetic ISED of clusters (regarded as the real SSP) are enhanced towards shorter wavelengths compared with the conventional models. This implies that a stellar population may look younger (bluer colors) or more metal-poor than the conventional SSP. In other words, if conventional SSP models are used to fit the ISED and measure the age or metallicity of a real stellar population, one or both parameters will be underestimated compared to the true parameters, thus posing a problem for our current understanding of galaxies.

We will show that the synthetic ISED can be fitted very well with conventional SSP models, but the uncertainties are significant. Shown in Figure 1 is an ISED fit to NGC 2420 using conventional SSP models, by either fixing the metallicity to obtain the age (left), or fixing the age to derive the metallicity (right). It is clear that good-quality fits (much better than for unresolved observations) yield nevertheless very different ages or metallicities compared to the true parameters. This technique actually measures the uncertainties in age or metallicity in EPS.

\subsection{Uncertainty in age}

For a given set of parameters of age and metallicity, the synthesized ISED of a cluster is enhanced towards shorter wavelengths with respect to the conventional SSP models because of the inclusion of BSs. This enhancement is the cause of incorrect age determinations on the basis of conventional SSP model fits (XD05; XDH06). 

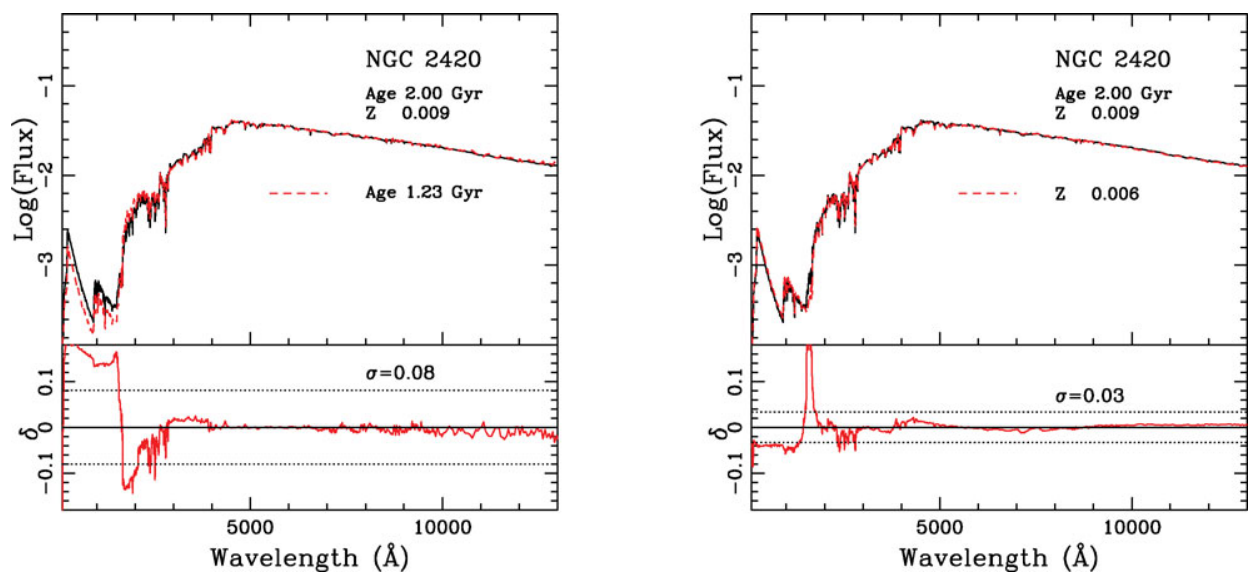

Figure 1. Examples of fits to the synthetic ISEDs of clusters using conventional SSP models. (left) Adopting the correct metallicity and leaving age as a free parameter. The original parameters of the clusters are given in the upper right-hand corner in each panel. The solid line is the synthetic ISED, while the dashed line represents the conventional SSP model. The lower part shows the fit errors. (right) As for the left panel, but for metallicity as a free parameter.

For clusters with relatively robust parameter determinations, the true age values versus those from conventional SSP fits are shown in the left panel of Figure 1. The distance from the diagonal represents the uncertainties in age for each cluster, shown as dots in the plot.

\subsection{Uncertainty in metallicity}

Figure 1 (right panel) shows fits of the synthetic ISEDs for the same clusters as in previous subsection using the conventional SSP models. In each panel, the original parameters of the clusters are included. The solid lines in the plots are for the synthetic ISED, while the dashed lines are for the fitted conventional SSPs (whose metallicities are also indicated). The lower part of each panel shows the fitting accuracy. As can be seen, the fits are very good. Across the entire range of optical bands, the difference is generally below a few percent, better than what observational photometry can actually distinguish. The only sizable deviation is found in the ultraviolet.

It is clear from Figure 2 that, when compared with age fits, the scatter in metallicity is much larger. The linear fit only shows the trend. One of the possible reasons for this would be the limited grid coverage in metallicity. There are only five metallicities in the library, which makes interpolation much less accurate than for our age fits.

\subsection{Uncertainty in mass-to-light ratio}

Due to the BS content in general stellar populations, the normal way of estimating their mass using photometric data will be affected. This can be shown on the basis of a comparison between the mass-to-light ratios (MLRs) of the synthetic with respect to the conventional SSP models. The mass of an SSP will not be affected by BS formation, because most BS formation processes conserve mass, at least approximately. Therefore, alterations to the MLR are due to changes in the luminosity of the SSP. In general, the formation of BSs will contribute more light to the system, since BSs form a more massive star from low-mass progenitors. However, this needs more investigation. 

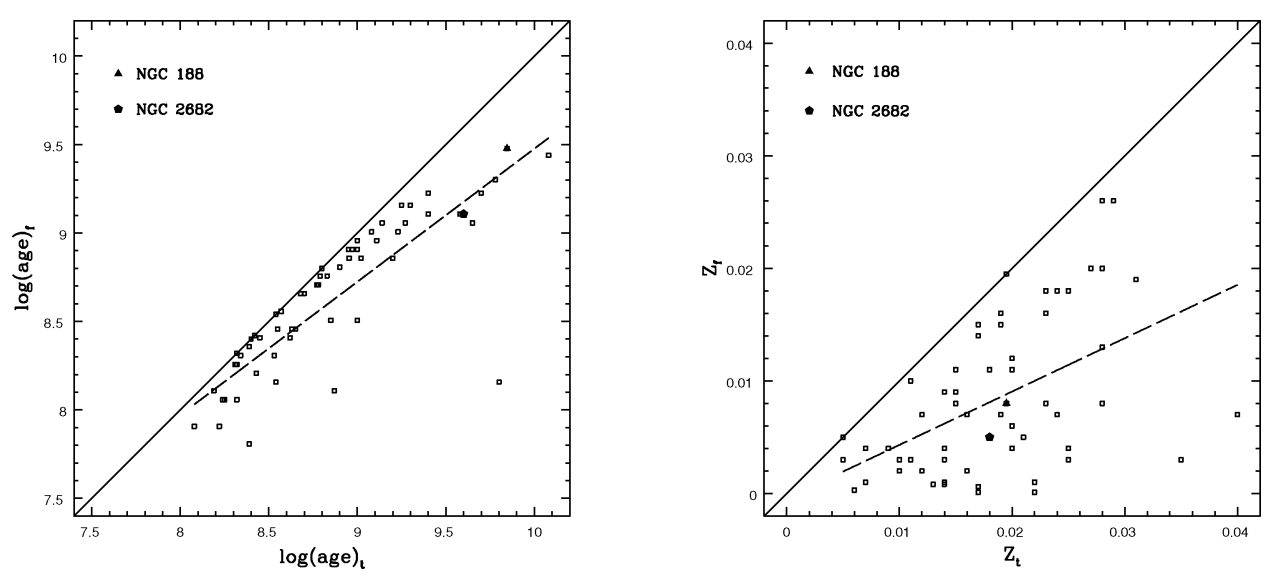

Figure 2. Synthetic age (left) and metallicity (right) of open clusters (vertical axis) versus isochrone-fitted values (horizontal axis). The two well-studied and relatively well-populated clusters M67 (NGC 2682) and NGC 188 are indicated by heavy dots. A linear fit to the sample clusters is given by the dotted line.

\section{Comparison between cluster ISEDs and binary synthesis}

Because the empirical method discussed above is limited by both age and metallicity coverage of the sample of stars clusters, constructing a complete library of revised SSP models is not possible at the present time. Further applications of observationally constrained SSP models to EPS need more effort and a larger, more diverse sample. Therefore, a theoretical approach to the problem becomes important if we want to reach a perfect solution. This will need to rely on statistical studies of the detailed physical processes, modeling of stellar evolution with all processes included, and hence will need a lot of effort.

Interacting binary systems are believed to be one of the major ways of BS formation. There are two channels of BS formation related to interactions in close binaries, mass exchange between the components (Zhang et al. 2004, 2005; Tian et al. 2006; Lu et al. 2009) and coalescence (Chen \& Han 2009), which will eventually happen in some close binary systems. Thanks to recent advances in studies of binary evolution, population synthesis involving binaries ('binary synthesis') can be done. This provides another way of getting a more realistic picture of SSPs than the conventional scenario (Hurley et al. 2005; Tian et al. 2006; Zhang et al. 2005) and can be regarded as a partial solution to the current problem.

To explore the capability of binary evolutionary models and the binary synthesis technique, we compare the results of Zhang et al. (2005) to the conventional SSP models from BC03, in terms of broad-band colors derived from SEDs of both datasets. Figure 3 (left) shows that, due to the formation of BSs with the assumptions made for the binary parameters in a population, the binary population has bluer colors for almost all ages (except for part of $V-I$ ) with respect to BC03. Just like the synthetic ISED of a star cluster, the ISED of a binary population is enhanced at shorter wavelengths compared with the conventional SSP model, as shown in Figure 3 (left). This enhancement is systematic in the model grid (see Zhang et al. 2004).

A comparison with our empirical approach is shown in the right-hand panel of Figure 3. If we derive the age using the above-mentioned technique, both binary synthesis (dashed line) and the empirical ISED (open dots; size proportional to fitting error) predict a 

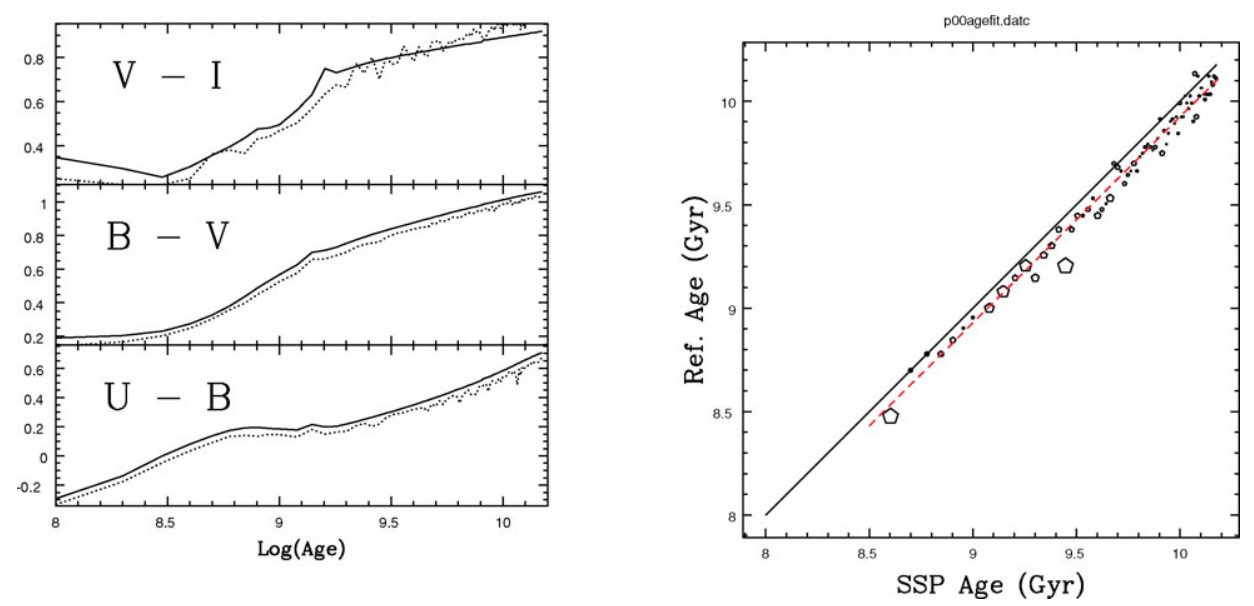

Figure 3. (left) Broad-band colors derived from binary synthesis (dotted lines) compared with the conventional BC03 model (solid lines). The ages predicted by binary synthesis are systematically younger than resulting from the conventional model (right) (dashed line) due to the bluer colors. The synthetic ISEDs of the clusters are shown as open symbols (size proportional to fitting errors), showing not only a large scatter due to stochasticity, but also younger ages, on average, than predicted by binary synthesis.

younger age. It is clear that the theoretical results from binary population models cannot fully explain the age discrepancy due to the existence of BSs in star clusters. We anticipate that, instead of considering binary mass exchange alone, a full consideration of all BS formation channels in clusters may reach closure with regard to this problem. Although not perfect in covering parameter space and all possible evolutionary phases, stellar population models incorporating close binary systems can, at least in part, reveal the effects of remnants (mostly BSs) on conventional SSP approaches. Bearing in mind that collisional encounters and mergers are not involved, the results presented here on the basis of binary synthesis should be regarded as a lower limit to the modifications to the conventional SSP models.

\section{Discussion and concluding remarks}

We have reviewed the role of BSs in star clusters and the contribution of BSs to the integrated light of the host stellar populations based on a sample of Galactic open clusters.

Considered as a general case for stellar populations covered by the parameter range of our OC sample, the uncertainties in the conventional SSP model due to neglecting stellar interactions are measured in terms of the basic parameters. It is shown that the results of EPS for the populations represented by the OC sample differ significantly from the correct values. If age and metallicity are both uncertain because of other problems (Charlot et al. 1996), the MLR will also be highly uncertain. If one uses the conventional SSP models, the mass of a population estimated based on $V$-band data, can be overestimated by as much as $20 \%$ based on our OC analysis. However, considering binary population synthesis, deriving the correct MLR is more complicated. BS formation in a stellar population from binary mass exchange usually involves a star near the turnoff. The high-luminosity red giant-branch (RGB) phase following the single-star evolutionary path will thus be avoided. As a result, the population gains light in the blue (BS contribution) and loses 


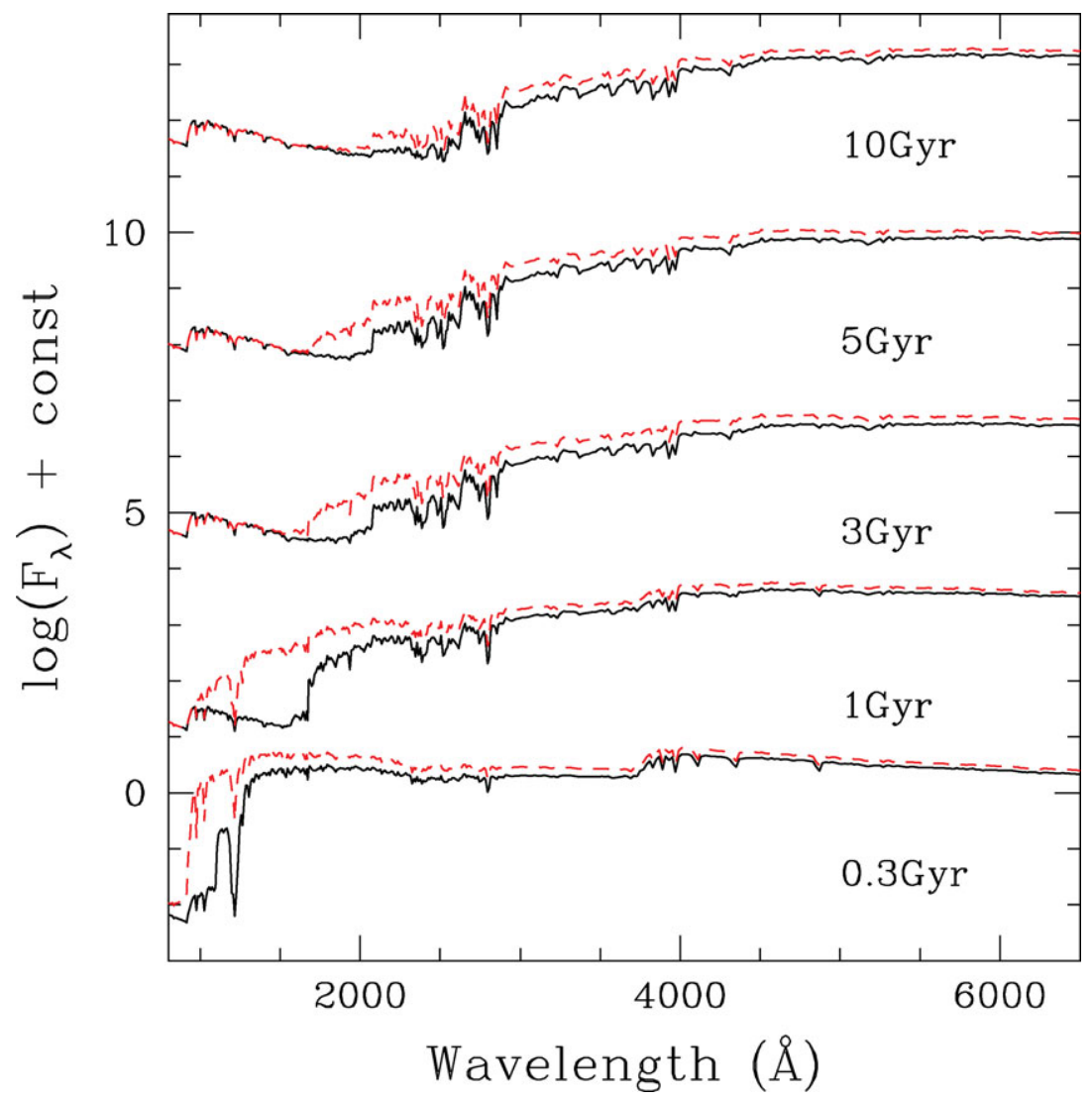

Figure 4. Comparison between BS03 ISEDs of SSP models (lower solid spectra) and the empirically revised SSP models with the same ages (indicated for each pair of ISEDs) for solar metallicity.

light in the red (avoiding the RGB phase). Therefore, the bolometric luminosity of a population may be reduced because of BS formation (Chen \& Han 2009).

Using photometric observations of Galactic OCs (Ahumada \& Lapasset 1995), we have tested the modifications to the main properties of the conventional SSP model and its uncertainties in applications of population analysis. We have shown that, limited to the age and metallicity parameters of the OC sample, the conventional SSP models are seriously affected, and this may have severe implications for galaxy population studies using EPS. To correct for these errors, studies of SSPs using star clusters of a larger sample and using the binary synthesis method with all interacting processes taken into account are both needed. Work to generate modified SSP models based on our current results is in progress (see for preliminary results Figure 4).

\section{Acknowledgements}

We thank the Chinese National Science Foundation for partial support through grants 10973015, 10573022 and 10778719, and the Ministry of Science and Technology of China through grant 2007CB815406. We would like to thank Z. Han, F. H. Zhang and X. F. Chen for providing binary population synthesis models. 


\section{References}

Ahumada, J. \& Lapasset, E. 1995, A\&AS, 109, 375 (AL95)

Bertelli, G., Bressan, A., Chiosi, C., Fagotto, F., \& Nasi, E. 1994, A\&AS, 106, 275

Bica, E. \& Alloin, D. 1986a, A\&SAS, 66, 171

Bica, E. \& Alloin, D. 1986b, A\&A, 162, 21

Bressan, A., Chiosi, C., \& Fagotto, F. 1994, ApJS, 94, 63

Bruzual G. \& Charlot, S. 2003, MNRAS, 344, 1000 (BC03)

Charlot, S. Worthy, G., \& Bressan, A. 1996, ApJ, 457, 625

Chen, X., \& Han, Z.W. 2009, MNRAS, 395, 1822

Deng, L., Chen, R., Liu, X. S., \& Chen, J. S. 1999, ApJ, 524, 824

Dias, W. S., Alessi, B. S., Moitinho, A., \& Lépine, J. R. D. 2002, A $\& A, 389,871$

Ferraro, F. R., Sills, A., Rood, R. T., Paltrinieri, B., \& Buonanno, R. 2003, ApJ, 588, 464

Hurley, J. R., Pols, O. R., Aarseth, S. J., \& Tout, C. A. 2005, MNRAS, 363, 293

Liu, G. Q., Deng, L., Chávez, M. D., Bertone, E., Herrero, D. A., \& Mata-Chávez, M. D. 2008, MNRAS, 390, 665

Piotto, G., De Angeli, F., King, I. R., Djorgovski, S. G., Bono, G., Cassisi, S., Meylan, G., Recio-Blanco, A., Rich, R. M., \& Davies, M. B. 2004, ApJ (Letters), 604, L109

Sandage, A. R. 1953, AJ, 58, 61

Sills, A. I., Adams, T., \& Davies, M. B. 2005, MNRAS, 358, 716

Worthey, G. 1994, ApJS, 95, 107

Xin, Y. \& Deng, L. 2005, ApJ, 619, 824 (XD05)

Xin, Y., Deng, L., \& Z. Han 2007, ApJ, 660, 319 (XDH06)

Zhang, F., Han, Z., Li, L., \& Hurley, J. R. 2004, A\& A, 415, 117

Zhang, F., Li, L., \& Han, Z. 2005, MNRAS, 364, 503 